\title{
Improvement of Nonrigid-Earth Nutation Theory by Adding a Model Free Core Nutation Term
}

\author{
Toshimichi Shirai \\ University of Tokyo, Graduate School of Science, Department of \\ Astronomy, 3-8-1, Hongoh, Bunkyo-ku, Tokyo 113-0033, Japan \\ t.shirai@nao.ac.jp \\ Toshio Fukushima \\ National Astronomical Observatory, \\ 2-21-1, Ohsawa, Mitaka, Tokyo 181-8588, Japan
}

\begin{abstract}
From the analysis of VLBI observational data compiled by USNO (U.S. Naval Observatory) from MJD 44089.994 to 51618.250 (McCarthy, 2000), we showed that a strong peak around -400 sidereal days in the spectrum of its differences from the IERS96 nutation theory could be explained by adding a model Free Core Nutation (FCN) term in the form of a single damped oscillation. Then we developed a new analytical theory of the nonrigid-Earth nutation including the derived FCN model. We adopted RDAN98 (Roosbeek and Dehant, 1998) as the rigid Earth nutation theory. It was convolved with a transfer function using numerical convolution in the time domain (Shirai and Fukushima, 2000). The form of the transfer function was the same as that of Herring (1995). However, its free parameters such as the complex amplitude and frequency of the FCN were readjusted by fitting to the above VLBI data. Even after truncating the forced nutation series so as to contain only 180 terms, the WRMS (Weighted Root Mean Square) of the complex residuals for the new nutation series is 0.312 mas, which is significantly smaller than 0.325 mas, that of the IERS96 nutation theory. As for the FCN term, we estimated its oscillatory period as $-430.8 \pm 0.6$ sidereal days, and its $Q$-value as $16200 \pm 1600$. Also we estimated the correction of the precession constants as $-0.29297 \pm 0.00047^{\prime \prime} / \mathrm{cy}$ in longitude and $-0.02430 \pm 0.00019^{\prime \prime} / \mathrm{cy}$ in obliquity, respectively.
\end{abstract}

\section{Introduction}

The nutation has two modes, the forced and the free modes. The forced nutation is due to the lunisolar and planetary tidal forces. The free mode appears because the Earth has a rotating and ellipsoidal fluid core. Thus this mode was named as the free core nutation (FCN) by Toomre (1974). However the current IAU nutation theory, which is called the IAU1980 nutation theory (Seidelmann, 1982), does not include the FCN term. This is because, at the time, the FCN had not yet been observed directly optically (Sasao and Wahr, 1981). 
The IERS96 nutation theory, which is in the best agreement with the VLBI observation, also does not contain the FCN term (McCarthy, 1996). See Figure 1 for the difference between the VLBI observation and IERS96 nutation theory for the period MJD 44089.994 to 51618.250 . There remains a strong peak around -400 sidereal days in its spectrum, shown in Figure 2. This is probably because the strong peak in the spectrum of the residuals is believed to be explained by considering external excitations which vary with the periods close to that of the FCN. As shown by Gegout et al. (1998), the atmosphere is a good candidate for such an exciting force, which means that the amplitude of such peaks cannot be predicted. However, the strong peak may be explained appropriately by adding a model FCN term. In this paper, we will try to improve the analytical theory of the nonrigid-Earth nutation by adding a simple model term of the FCN.

\section{Model of Free Core Nutation}

We first fixed the forced nutation theory as the IERS96 nutation theory. We set the frequency of the FCN as that of Herring (1995). As for the model of FCN, we considered the following two: (A) no excitation during the observation (model A), and (B) frequent excitations during the observation (model B). Model A is mathmatically expressed as a single damped oscillation, where the damping is due to the viscosity of the outer fulid core and the magnetic coupling of mantle-core boundary. Model B is regarded as a simple harmonic oscillation without damping whose (real) frequency $\omega$ is close to the real part of the FCN frequency. In both models, we estimated one complex amplitude of the FCN by weighted least-squares fitting to the residuals. In model $\mathrm{B}$, we also derived the real frequency $\omega$ by fitting. In both models, the strong peak in the spectrum around -400 sidereal days is clearly removed by adding a model FCN term. See Figure 2 for model A. From the analysis of WRMS (Weighted Root Mean Square) and spectrum and number of parameters, we concluded that model A (WRMS $=0.3030$ mas) is better than the model $\mathrm{B}$ (WRMS $=0.3051$ mas). In conclusion we adopted the model of the FCN term as

$$
\begin{gathered}
\Delta \psi_{\mathrm{FCN}} \sin \epsilon_{0}=e^{-\omega_{\mathrm{FCN}}^{i} t}\left(C_{\mathrm{FCN}}^{r} \cos \omega_{\mathrm{FCN}}^{r} t-C_{\mathrm{FCN}}^{i} \sin \omega_{\mathrm{FCN}}^{r} t\right) \\
\Delta \epsilon_{\mathrm{FCN}}=e^{-\omega_{\mathrm{FCN}}^{i} t}\left(C_{\mathrm{FCN}}^{i} \cos \omega_{\mathrm{FCN}}^{r} t+C_{\mathrm{FCN}}^{r} \sin \omega_{\mathrm{FCN}}^{r} t\right)
\end{gathered}
$$

\section{Method}

We developed a new nutation series by incorporating the above model FCN term. Usually the theory of the forced nutation is obtained as a combination of the rigid-Earth nutation theory and the transfer function of the nonrigid Earth. As the former, we adpopted the RDAN98 (Roosbeek and Dehant, 1998). Then we removed from RDAN98 the periodic terms whose periods are longer than 150 years. As the transfer function, we selected the same functional form as that of Herring (1995);

$$
g(\hat{\omega})=R+R^{\prime} \hat{\omega}+\frac{R_{\mathrm{FCN}}}{\hat{\omega}-\hat{\omega}_{\mathrm{FCN}}}+\frac{R_{\mathrm{CW}}}{\hat{\omega}-\hat{\omega}_{\mathrm{CW}}}
$$




\section{Residual of Each Series}

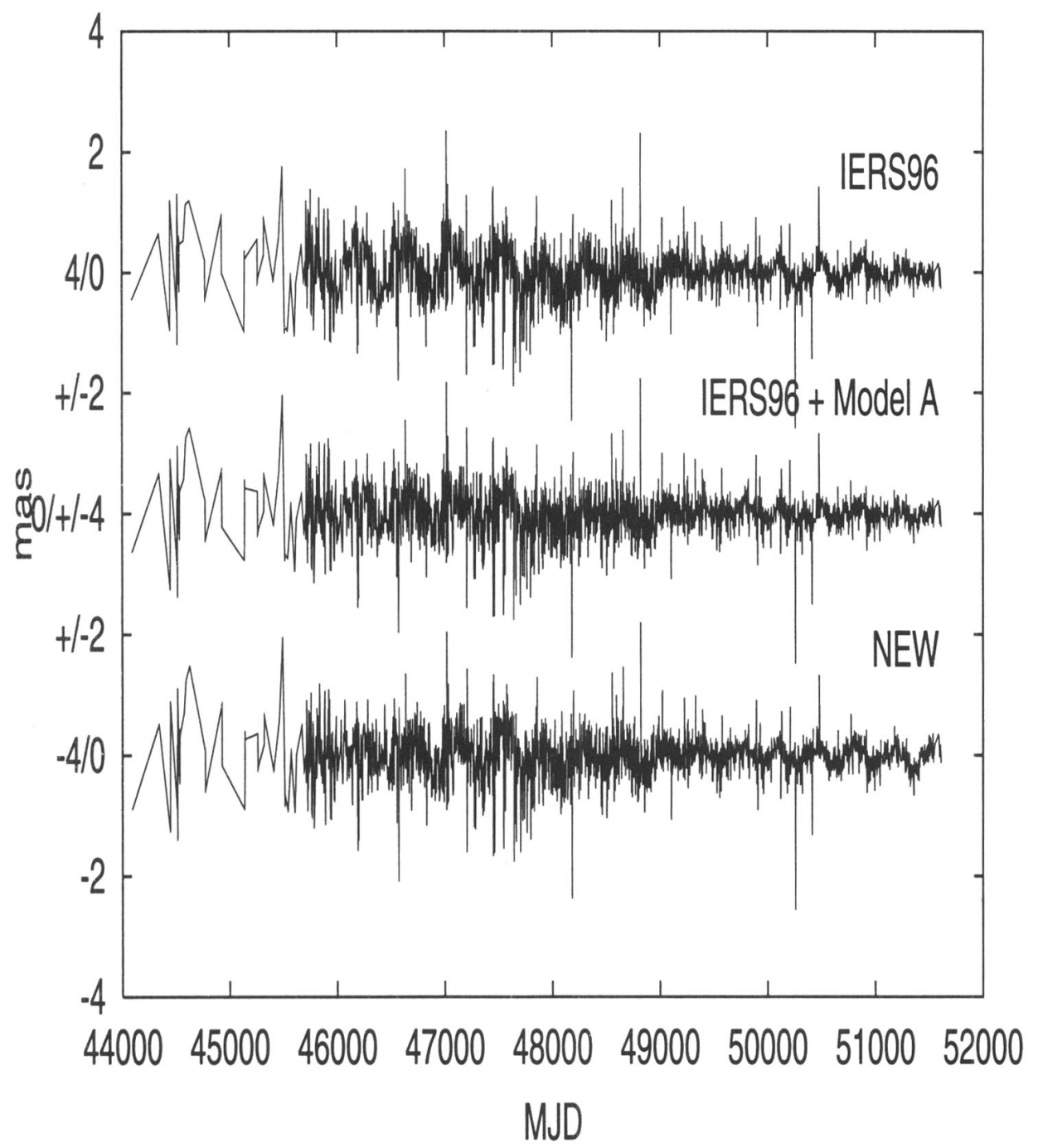

Figure 1. Residuals of IERS96 Nutation Theory and Model A and New Series. Shown are the differences between the VLBI data and each series. 

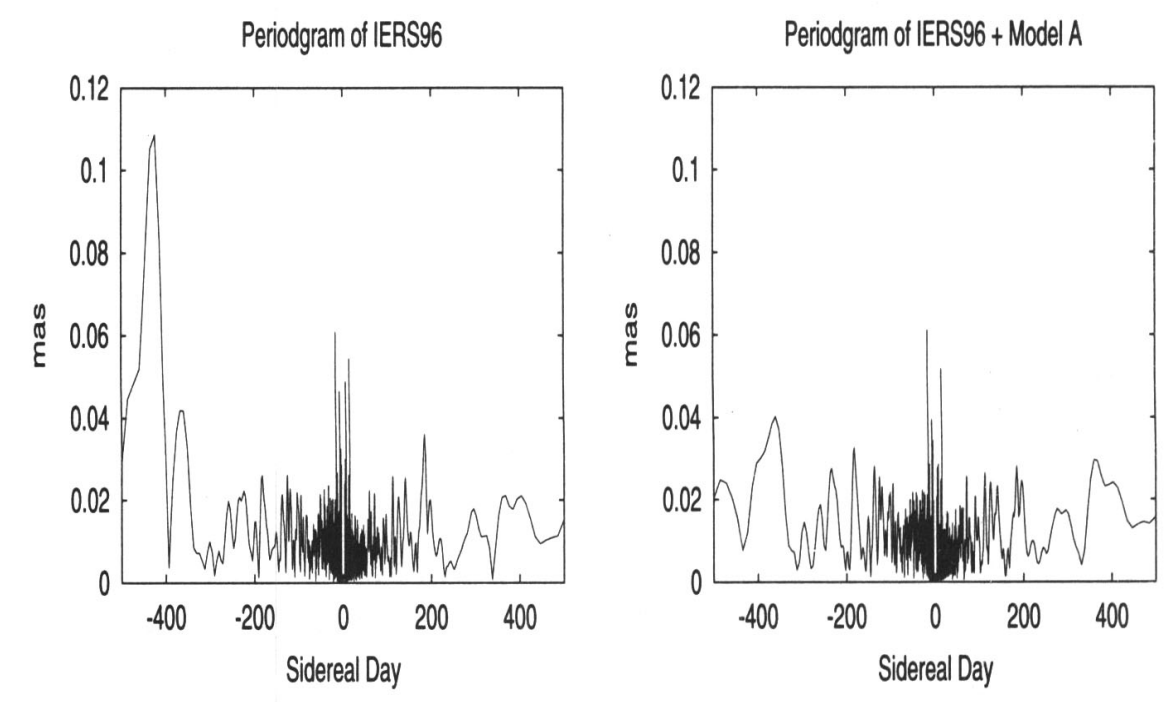

Figure 2. Spectra of Complex Residuals for IERS96 Nutation Theory with and without FCN. The strong peak in the spectrum of the IERS96 nutation theory around -400 sidereal days is clearly removed by adding a model FCN term.

where $g(\hat{\omega})$ is the ratio of the nutation amplitude of the nonrigid Earth, $\hat{\omega}$ is the frequency normalized by the mean angular velocity of the Earth rotation $\Omega_{0}$ as $\hat{\omega}_{\mathrm{FCN}}=\omega_{\mathrm{FCN}} / \Omega_{0}$, where $\Omega_{0}=7.292115 \times 10^{-5} \mathrm{rads}^{-1}$. Here we ignored the contribution of the inner solid core of the Earth. We convolved the transfer function with the nutation theory of the rigid Earth by numerical convolution in the time domain (Shirai and Fukushima, 2000). We used the VLBI data compiled by USNO for the period MJD 44089.994 to 51618.250 . We removed the geodesic nutation discussed by Fukushima (1991) from the VLBI data before analysis. We fixed $R_{\mathrm{CW}}$ and $\hat{\omega}_{\mathrm{CW}}$ at their geophysically estimated values. We also included in the parameters to be fitted the correction to the IAU1976 precession formula (Lieske et al., 1977), namely the offsets and rates of nutation. We included the complex amplitude of FCN, $C_{\mathrm{FCN}}=C_{\mathrm{FCN}}^{r}+i C_{\mathrm{FCN}}^{i}$ in the parameters to be fitted. In summary, we simutaneously estimated seven complex parameters comprised of (1) $R, R^{\prime}, R_{\mathrm{FCN}}$, and $\hat{\omega}_{\mathrm{FCN}},(2)$ two complex ones corresponding to the offsets and rates of nutations in longitude and in obliquity, and (3) the complex amplitude of the FCN, $C_{\mathrm{FCN}}$. The estimation was done by the weighted nonlinear least-squares method. This approach is almost the same as that used to create the IERS96 nutation theory. The main differences are in (1) the FCN term which we included while Herring did not, (2) the additional 

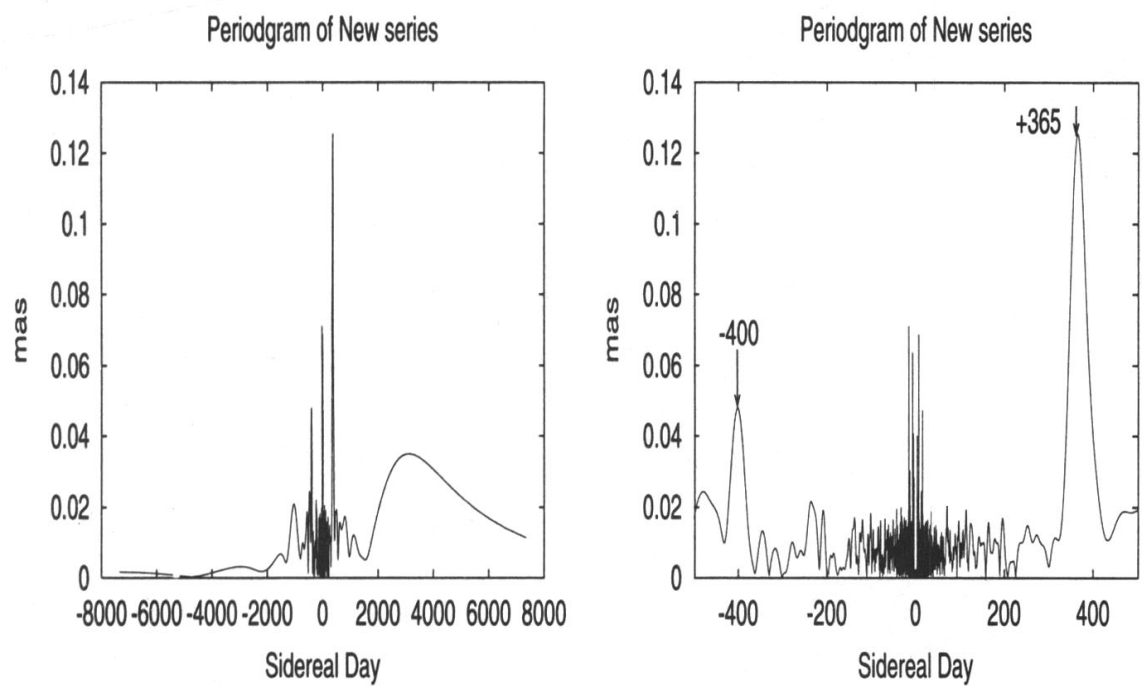

Figure 3. Spectra of complex residuals for new series.

annual prograde term which Herring estimated while we did not, and (3) the period of VLBI observation.

\section{Results and Discussion}

The WRMS of the residuals for the new series is 0.3075 mas, which is about $6 \%$ smaller than 0.325 mas of the IERS96 nutation theory. The estimated parameters are listed in Table 1. Figure 1 shows the residuals for the new series. Figure 3 shows the periodgram of the new nutation series for the prograde and the retrograde bands. The original RDAN98 contains 1501 terms. After convolution with the transfer function, we sorted the series in amplitude and kept only the top 180 terms. This is because the WRMS of 0.3120 mas was only $1.5 \%$ larger than the 0.3075 mas value of the full series. See Figure 4 for the number-of-terms dependence of WRMS and amplitude. Clearly the RMS does not siginificantly decrease after the number of terms exceeds 180 . On the other hand, the number-of-terms dependence of the new series decreases linearly in a $\log$-log plot. In summary, we consider the truncated series is precise enough for practical purposes. The full paper will be submitted to Astron. J. soon. 
Table 1. Estimated Parameters

\begin{tabular}{lrr} 
& Real part & Imaginary part \\
\hline$R$ & $1.049018 \pm 0.000011$ & $0.001560 \pm 0.000011$ \\
$R^{\prime}$ & $-0.2570 \pm 0.0007$ & $-0.0406 \pm 0.0007$ \\
$R_{\mathrm{FCN}}$ & $(-1.1499 \pm 0.0002) \times 10^{-4}$ & $(-2.17 \pm 0.02) \times 10^{-6}$ \\
$\hat{\omega}_{\mathrm{FCN}}$ & $(-2.3206 \pm 0.0026) \times 10^{-3}$ & $(3.06 \pm 0.26) \times 10^{-5}$ \\
$C_{\mathrm{FCN}}[\mu a s]$ & $-78 \pm 3$ & $-0.6 \pm 3$ \\
\cline { 2 - 3 }$\Delta \psi$ & Offset [mas] & Rate [mas $/ \mathrm{yr}]$ \\
\cline { 2 - 3 }$\Delta \epsilon$ & $-42.458 \pm 0.033$ & $-2.9297 \pm 0.0047$ \\
$\Delta \epsilon$ & $-5.186 \pm 0.013$ & $-0.2430 \pm 0.0019$ \\
\hline
\end{tabular}
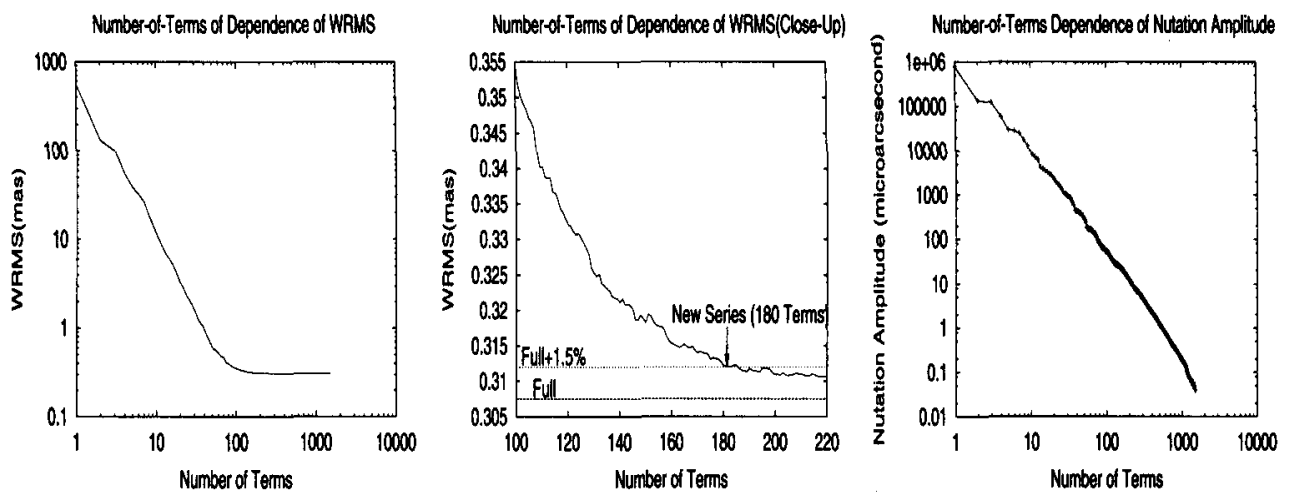

Figure 4. Number-of-terms dependence of WRMS and nutation amplitude. 


\section{References}

Dehant, V., et al., 1999, Celest. Mech. Dyn. Astron., 72, 245.

Fukushima, T., 1991, Astron. Astrophys., 244, L11.

Gegout, P. et al., 1998, Phys. Earth. Planet. Inter., 106, 337

Herring, T. A., 1995, Hightlights of Astronomy, 10, 222.

Lieske, J. H., Lederle, T., Fricke, W., and Morando, B., 1977, Astron. Astrophys., $\mathbf{5 8}, 1$.

McCarthy, D. D., 1996, 'IERS Conventions', IERS Technical Note 21.

McCarthy, D. D., 2000, private communication.

Roosbeek, F. and Dehant, V., 1998, Celest. Mech. Dyn. Astron., 70, 215.

Sasao, T. and Wahr, J. M., 1981, Geophys. J. R. Astron. Soc., 64, 729.

Seidelmann, P. K., 1982, Celest. Mech., 27, 79.

Shirai, T. and Fukushima, T., 2000, Astron. J., 119.

Toomre, A., 1974, Geophys. J. R. Astron. Soc., 38, 335. 